Original Article

\title{
An experience on the model-based evaluation of pharmacokinetic drug-drug interaction for a long half-life drug
}

\author{
Yunjung Hong ${ }^{1,2}$, Sangil Jeon ${ }^{3}$, Suein Choi ${ }^{1,2}$, Sungpil Han ${ }^{1,2}$, Maria Park ${ }^{1,2}$, and Seunghoon Han ${ }^{1,2 * *}$ \\ ${ }^{1}$ PIPET (Pharmacometrics Institute for Practical Education and Training), College of Medicine, The Catholic University of Korea, ${ }^{2}$ Department of Pharmacology, \\ College of Medicine, The Catholic University of Korea, ${ }^{3} Q$-fitter, Inc., Seoul 06591, Korea
}

\section{ARTICLE INFO}

Received April 23, 2021

Revised August 10, 2021

Accepted September 7, 2021

*Correspondence

Seunghoon Han

E-mail:waystolove@catholic.ac.kr

\section{Key Words}

Drug interactions

Half-Life

Model-based evaluation

Pharmacokinetics
ABSTRACT Fixed-dose combinations development requires pharmacokinetic drugdrug interaction (DDI) studies between active ingredients. For some drugs, pharmacokinetic properties such as long half-life or delayed distribution, make it difficult to conduct such clinical trials and to estimate the exact magnitude of DDI. In this study, the conventional (non-compartmental analysis and bioequivalence [BE]) and modelbased analyses were compared for their performance to evaluate DDI using amlodipine as an example. Raw data without DDI or simulated data using pharmacokinetic models were compared to the data obtained after concomitant administration. Regardless of the methodology, all the results fell within the classical BE limit. It was shown that the model-based approach may be valid as the conventional approach and reduce the possibility of DDI overestimation. Several advantages (i.e., quantitative changes in parameters and precision of confidence interval) of the model-based approach were demonstrated, and possible application methods were proposed. Therefore, it is expected that the model-based analysis is appropriately utilized according to the situation and purpose.

\section{INTRODUCTION}

Fixed-dose combinations (FDC) contribute to a better clinical outcome with increased compliance over monotherapy [1-3]. In this context, many pharmaceutical companies are interested in the development of FDC. Drug-drug interaction (DDI) studies between active ingredients are required in FDC development, which generally encompasses multiple-dose studies. However, such clinical trials may encounter difficulties due to some pharmacokinetic (PK) properties, e.g., long half-life and delayed distribution. Drugs with such properties may cause carry-over between dosing, resulting in long sampling periods and wash-out periods, or steady-state achievement may be questioned in studies designed to avoid prolonged clinical trials, e.g., one-sequence cross-over design.
A typical example is amlodipine. Amlodipine is a frequently prescribed drug for managing hypertension and coronary artery disease in people suffering from angina [4]. Various medications such as statins for hyperlipidemia and cardiovascular disease treatment, or angiotensin receptor blockers and thiazide-like diuretics for hypertension treatment are used concomitantly as combination therapy with amlodipine to enhance the efficacy in patients. Conventional non-compartmental analysis (NCA) for DDI evaluation for this kind of drug may not be suitable due to the possibility of incomplete attainment of full-PK concentrationtime profiles where $A U C_{\text {inf }}$ and terminal half-life $\left(t_{1 / 2}\right)$ are misestimated, producing inaccurate interpretations as a consequence. Therefore, several suggestions have been proposed to overcome NCA limitations regarding drugs with a long half-life [5-8].

Among these, model-based analysis has been recommended

\section{(i) (8)}

This is an Open Access article distributed under the terms of the Creative Commons Attribution Non-Commercial License, which permits unrestricted non-commercial use, distribution, and reproduction in any medium, provided the original work is properly cited. Copyright $\odot$ Korean J Physiol Pharmacol, pISSN 1226-4512, elSSN 2093-3827
Author contributions: Y.H., S.J., S.C., and S.H. performed the conception and design of the study; Y.H., S.C., M.P., S.P.H., and S.H. performed data acquisition; Y.H., S.J., and S.H. performed data analysis and interpretation; Y.H. and S.H. wrote and revised the manuscript; Y.H., S.C., and S.H. and discussed the results and commented on the manuscript. 
as an alternative method to NCA. Model-based analysis can comprehend the full concentration-time profiles even with sparse or incomplete data, allowing various scenarios to be simulated and can integrate prior information from the literature [6]. Also, Svensson et al. [7] reported that the NCA approach underpredicted DDI impact and showed bias in the bedaquiline (half-life more than five months) simulation study. Conversely, model-based analysis was unbiased and showed an increased precision in DDI predictions. The terminal half-life was estimated ten times lower in NCA than the actual value, caused by invalid extrapolation from insufficient sampling. The discrepancy between the two methods highlighted the accuracy of the model-based analysis compared to NCA [7]. Yet, the knowledge toward a comparison between strategies is incomplete and challenges should be clarified [9]. Further evaluation and research are needed for the utilization of the method.

The study's objective was to compare NCA and model-based analysis for DDI evaluation using amlodipine data from a human pharmacology study. The clinical trial was adequately designed according to the applicable regulations with sufficient sampling periods for steady-state achievement. We expect to contribute and accumulate our experiences by establishing a standard PK comparison method for drugs with a long half-life.

\section{METHODS}

\section{Subjects and dataset}

The PK data were obtained from a DDI study for FDC development, designed as an open-label, multiple-dose, single-sequence cross-over, comparative PK study. This study was conducted at Seoul St. Mary's Hospital under all applicable regulations and ethical principles (Institutional Review Board control number: KC20MDSF0055).

The included subjects were Korean male volunteers whose mean values for age, weight, and height were 30 years (range: 23-46 years), $72.0 \mathrm{~kg}$ (range: $56.2-83.9 \mathrm{~kg}$ ), and $175.3 \mathrm{~cm}$ (range: 164-191 cm), respectively. No subjects had any evidence of underlying disease or history that could affect the study, including abnormal aspartate transaminase, alanine transaminase, total bilirubin and total cholesterol, allergic history reaction to the investigational drugs (amlodipine and potential interacting medications), excessive caffeine, alcohol consumption, and smoking, or any criteria that were considered ineligible by the investigator. The demographics and laboratory test results are summarized in Table 1.

Since it was a single-sequence cross-over study, amlodipine PK data without DDI were obtained during Period 1 (day 0-day 8), and that with possible DDI were obtained during Period 2 (day 9-day 13). A daily $5 \mathrm{mg}$ dose of amlodipine was administered to each subject over the whole study period. Period 1 PK data was generated by a single sample on day 7 (pre-dose) and serial samples on day 8 (at pre-dose, 1, 2, 3, 4, 5, 6, 7, 8, 9, 10, 12, and 24 $\mathrm{h}$ after dosing). For Period 2, the same samplings were performed on days 12 and 13. Although the population PK modeling can handle sparse or incomplete full-PK data, only data from subjects who completed the PK sampling schedule ( $n=14,392$ observations) were used to minimize the magnitude of possible selection bias.

\section{Overall scheme for DDI evaluation}

DDI evaluation was performed in three methods.

1. Conventional approach: NCA and bioequivalence (BE) approach

The maximum plasma concentration observed $\left(C_{\max }\right)$, the time to reach $C_{\max }$ from the last dosing $\left(t_{\max }\right)$, the area under the plasma concentration-time curve during a dosing interval $\left(A U C_{\tau}\right)$, and the half-life $\left(t_{1 / 2}\right)$ were assessed for each period. NonCompart package (version 0.4.7, by Kyun-Seop Bae, 2020) in R (version 3.6.3; R Foundation for Statistical Computing, Vienna, Austria) was utilized in this procedure. The $A U C_{\tau}$ was calculated according to the linear trapezoidal rule [10]. The $t_{1 / 2}$ was estimated as the value of $0.693 / \lambda_{z}$, where $\lambda_{z}$ is the terminal elimination slope with the best determination coefficient from the linear regression of the last $\geq$ three observations. An average BE assessment between two periods was performed using the nlme package (version 3.1-152, by José Pinheiro et al., 2021) in R. The log-transformed geometric mean ratios of $C_{\max }$ and $A U C_{\tau}$ with $90 \%$ confidence intervals (CI) were estimated. The DDI presence was assessed by whether the geometric mean ratios of the parameters and their 90\% CI fell within the conventional BE range (0.8-1.25) [11].

\section{Partial Model-based approach (MB1)}

A simulated PK profile (for the planned time of Period 2) using the PK model built from Period 1 data was compared to the observed PK profiles of Period 2 under the same schema as the NCA and BE approach. For the simulations, the individual PK param-

Table 1. Subject demographics

\begin{tabular}{lc}
\hline \multicolumn{1}{c}{ Variables } & Mean (range) \\
\hline Age $(\mathrm{yr})$ & $30(23-46)$ \\
Weight $(\mathrm{kg})$ & $72.0(56.2-83.9)$ \\
Height $(\mathrm{cm})$ & $175.3(164-191)$ \\
Albumin $(\mathrm{g} / \mathrm{dl})$ & $4.6(4.2-5.0)$ \\
Body mass index $\left(\mathrm{kg} / \mathrm{m}^{2}\right)$ & $23.4(19.2-26.5)$ \\
Alanine transaminase $(\mathrm{IU} / \mathrm{L})$ & $29.7(9.0-54.0)$ \\
Aspartate transaminase $(\mathrm{IU} / \mathrm{L})$ & $23.5(15.0-36.0)$ \\
Serum creatinine $(\mathrm{mg} / \mathrm{dl})$ & $0.9(0.7-1.1)$ \\
Creatinine clearance $(\mathrm{mL} / \mathrm{min})$ & $122.2(90.5-175.6)$ \\
Glomerular filtration rate $(\mathrm{GFR}) *$ & $99.5(77.3-131.1)$ \\
$\quad\left(\mathrm{ml} / \mathrm{min} / 1.73 \mathrm{~m}^{2}\right)$ & \\
\hline *GFR was calculated using Modification of Diet in Renal Disease \\
equation.
\end{tabular}


eter values were fixed to reflect each subject's PK characteristics. This procedure was repeated 1,000 times, and the proportion of negative results for DDI was obtained.

\section{Full Model-based approach (MB2)}

Population PK model was developed using both Period 1 and 2 data, and possible DDI was investigated as the change of each PK parameter as follows:

$$
P_{\mathrm{i}, 2}=P_{\mathrm{i}, 1}+\mathrm{PRD} \cdot D P_{\mathrm{i}}
$$

where $P_{\mathrm{i}, 1}$ is the value of $\mathrm{i}^{\text {th }} \mathrm{PK}$ parameter in Period $1, P_{\mathrm{i}, 2}$ is the value of $i^{\text {th }}$ PK parameter in Period 2, $D P_{\mathrm{i}}$ is the quantitative difference between $i^{\text {th }}$ PK parameter according to the Period, and PRD is the indicator for Period (0 for Period 1 and 1 for Period 2). No period effect other than DDI was assumed since the actual period effect cannot be assessed in a single-sequence cross-over study.

For comparison, a similar NCA and BE approach was also utilized, and simulated steady-state PK profiles (on day 13) without DDI and with DDI (if it exists) were compared. The simulation was repeated 1,000 times, and the average BE results were summarized.

\section{General procedures for model development}

Nonlinear mixed-effects modeling was conducted using NONMEM version 7.4 (Icon Development Solutions, Ellicott City, MD, USA). The model was developed by evaluating different absorption models, adopting a two-compartment model with firstorder absorption and first-order elimination initially [12]. The first-order conditional estimation with interaction (FOCE-I) was employed throughout the process. The most appropriate model selection was based on objective function values (OFV) evaluation, diagnostic plots (goodness-of-fit and individual plot) visual inspection, percent relative standard errors (\%RSE), and parameter estimates of population fixed- and random- effects. The model was considered more appropriate if a decrease in the OFV was more than 3.84 (p-value $<0.05, d f=1$ ) using the likelihood-ratio test. $\mathrm{R}$ was used for graphical analysis and model diagnostics.

Inter-individual variability of each parameter was described exponentially as:

$$
P_{\mathrm{ij}}=\theta_{\mathrm{i}} \cdot \exp \left(n_{\mathrm{ij}}\right)
$$

Where $P_{\mathrm{ij}}$ is the estimated parameter value for the $j^{\text {th }}$ individual, $\theta_{\mathrm{i}}$ is the typical value of the $i^{\text {th }}$ PK parameter, and $n$ is the betweensubject variability (BSV), following a normal distribution $\mathrm{n} \sim N(0$, $\left.\omega^{2}\right)$. Covariance between inter-individual variability was evalu-
A

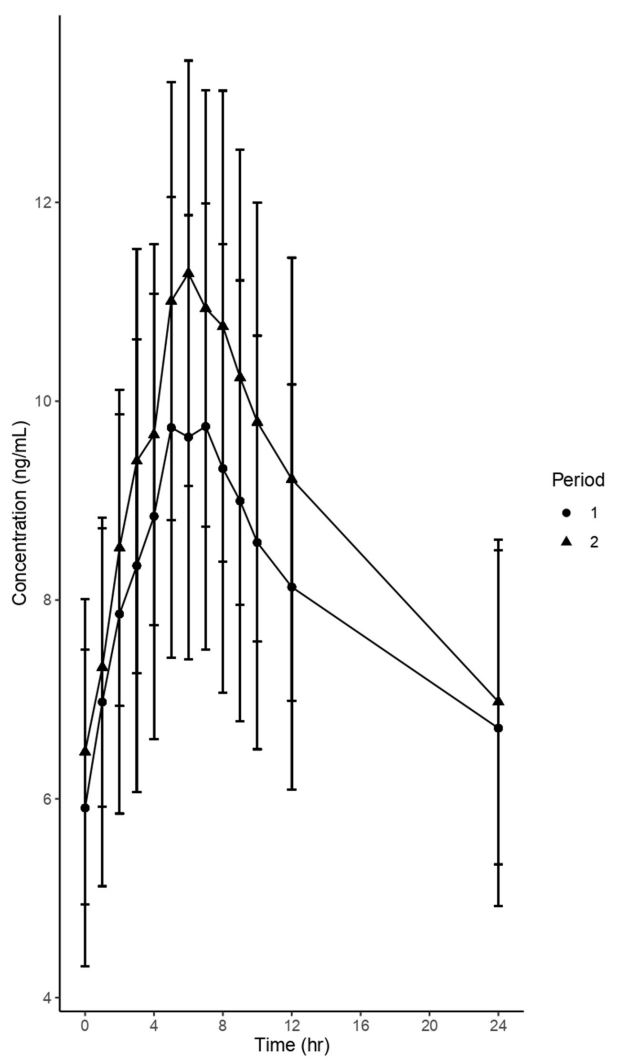

B

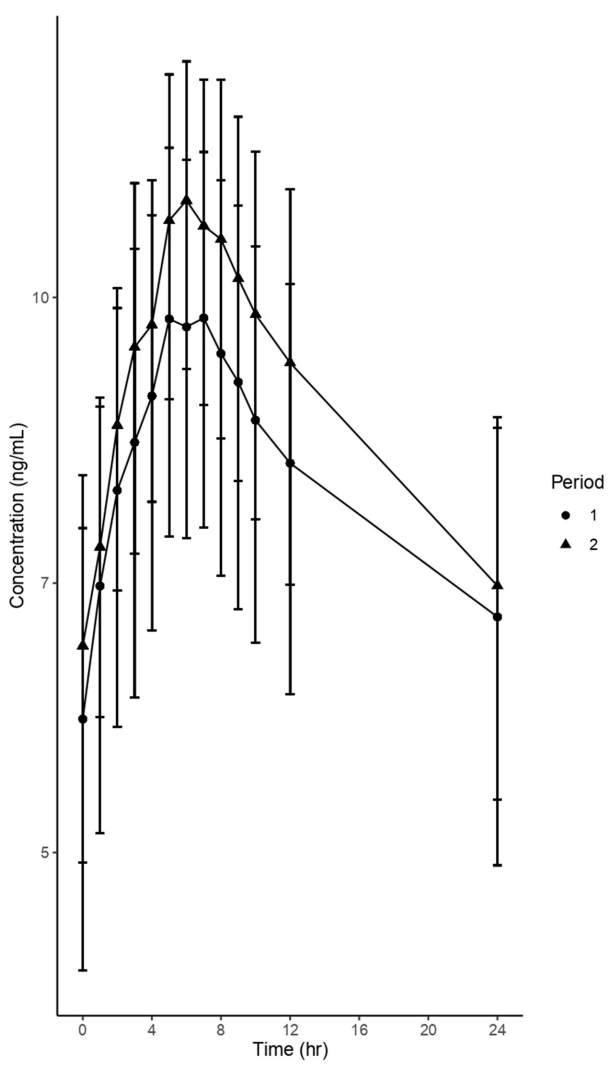

Fig. 1. Average plasma concentration versus time plots of amlodipine. (A) Linear scale, (B) Semilogarithmic scale. 
ated for possible correlations. Intra-individual variability (residual error) was tested with either additive, proportional, and combined structures.

\section{General procedures for model evaluation}

Visual predictive checks (VPCs) were conducted to support model adequacy. The 5th, 50th and 95th percentiles of the simulated data ( $\mathrm{n}=1,000$ replicates) were plotted, and the observed data were superimposed for visual comparison using $R$.

Wings for NONMEM (version 741, by Nick Holford, 2017) was used for bootstrap analysis to examine model robustness. One thousand replicates of the original dataset were generated by resampling with replacement, and median values with 95\% CIs of parameters were determined to compare with their respective final parameter values.

\section{RESULTS}

\section{Conventional approach (NCA \& BE)}

The $C_{\max }$ and $A U C_{\tau}$ values for Period 1 and 2 were $10.00 \pm 2.3$ $\mathrm{ng} / \mathrm{ml}$ and $11.69 \pm 2.38 \mathrm{hr} \cdot \mathrm{ng} / \mathrm{ml}$, and $192.46 \pm 47.35 \mathrm{ng} / \mathrm{ml}$ and $213.41 \pm 45.2 \mathrm{hr} \cdot \mathrm{ng} / \mathrm{ml}$, respectively. The observed average concentration-time profile is illustrated in Fig. 1 ([Fig. 1A] linear and [Fig. 1B] semilogarithmic), and PK parameters are summarized in Table 2. The point estimate for log-transformed geometric mean ratio of $C_{\max }$ was 1.177 (1.127-1.229), and that of $A U C_{\tau}$ was $1.119(1.068-1.174)$. The results fell within the BE limits of 0.8 to 1.25 , indicating there was no significant DDI.

\section{MB1}

A two-compartment, dual (rapid zero-order and slow firstorder) absorption followed by first-order elimination was chosen as the final structural model (Fig. 2). The estimated oral PK parameters and their values were as follows; clearance $(C L / F)=25.6$ $\mathrm{L} / \mathrm{h}$, central volume $\left(V_{2} / F\right)=884 \mathrm{~L}$, peripheral volume $\left(V_{3} / F\right)=$ $446 \mathrm{~L}$, intercompartmental clearance $(Q / F)=63.6 \mathrm{~L} / \mathrm{h}$, absorption rate constant $\left(K_{\mathrm{a}}\right)=0.874$, lag time for first-order absorption $\left(A L A G_{1}\right)=3.75 \mathrm{~h}$, duration of zero-order absorption $\left(D_{2}\right)=3.31 \mathrm{~h}$,

Table 2. Pharmacokinetic parameters using non-compartmental analysis

\begin{tabular}{lcc}
\hline \multicolumn{1}{c}{ Parameter } & Period 1 & Period 2 \\
\hline$C_{\max }(\mathrm{ng} / \mathrm{ml})$ & $10.00 \pm 2.3$ & $11.69 \pm 2.38$ \\
$A \cup C \tau(\mathrm{ng} \cdot \mathrm{hr} / \mathrm{ml})$ & $192.46 \pm 47.35$ & $213.41 \pm 45.2$ \\
$t_{1 / 2}(\mathrm{hr})$ & $46.67 \pm 31.6$ & $41.92 \pm 46.65$ \\
$t_{\max }(\mathrm{hr})$ & $5.5(5-8)$ & $6(5-9)$ \\
\hline
\end{tabular}

Data presented as mean \pm SD except for $t_{\max }$ values as median (range). and fraction absorbed through first-order absorption $\left(F_{1}\right)=0.458$. BSVs for $C L, V_{2}$, and $F_{1}$ and a correlation between $C L$ and $V_{2}$ were identified. Details for parameter estimates are shown in Table 3 with the bootstrap results and the goodness-of-fit plots illustrated in Fig. 3A. The model's predictive performance was judged by the VPC plot for Period 1 (Fig. 3B).

The point estimate for log-transformed geometric mean ratios of $C_{\max }$ and $A U C_{\tau}$ were 1.149 (1.097-1.203) and 1.105 (1.048-1.166), respectively. The results also proved that there was no significant DDI.

\section{MB2}

The model was best described by the same structure as the final model of MB1 (Fig. 2). DDI effect was explored for each PK parameter using the period as a covariate. Total bioavailability (BIO) was introduced as a relative bioavailability for Period 1. BIO showed a $30 \%$ increase compared to non-interacting situations, and $F 1$ and $C L$ showed approximately $16 \%$ and $15 \%$ increase, respectively. Estimated PK parameters and their values were as follows; clearance $(C L / F)=25.5 \mathrm{~L} / \mathrm{h}$, change in $C L=3.90 \mathrm{~L} / \mathrm{h}$, central volume $\left(V_{2} / F\right)=943 \mathrm{~L}$, peripheral volume $\left(V_{3} / F\right)=403 \mathrm{~L}$, intercompartmental clearance $(Q / F)=47.6 \mathrm{~L} / \mathrm{h}$, absorption rate constant $\left(K_{\mathrm{a}}\right)=0.991$, lag time for first-order absorption $\left(A L A G_{1}\right)$ $=3.86 \mathrm{~h}$, duration of zero-order absorption $\left(D_{2}\right)=3.42 \mathrm{~h}$, fraction absorbed through first-order absorption during Period 1 ( $F_{1}$ $(\mathrm{PRD} 1))=0.405$, fraction absorbed through first-order absorption during Period $2\left(F_{1}(\mathrm{PRD} 2)\right)=0.468$, and total bioavailability for Period $2(B I O)=1.30$. BSVs of $C L, V_{2}, F$, and $B I O$ were incorporated in the final model with a correlation between $C L$ and $V_{2}$. The final PK parameter estimates and bootstrap results are also presented in Table 3. The goodness-of-fit plots and VPC plots presented in Fig. 4A and 4B demonstrated that the model provided a sufficient description of the observed data.

The point estimate for log-transformed geometric mean ratios of $C_{\max }$ and $A U C_{\tau}$ were $1.139(1.092-1.188)$ and $1.116(1.064-1.170)$,

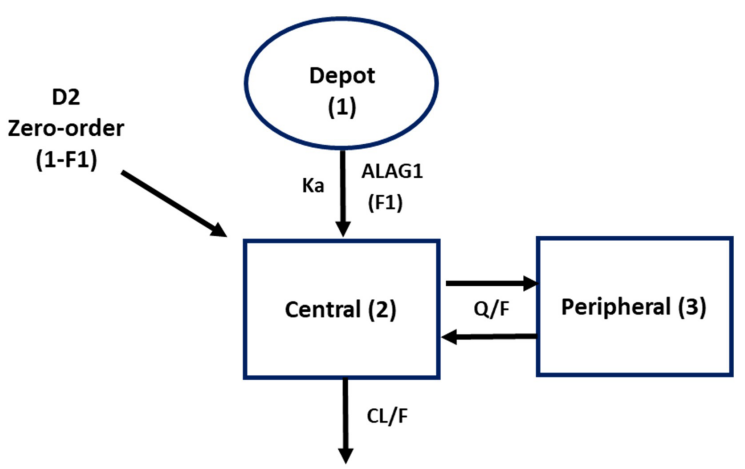

Fig. 2. PK Model structure. $D_{2}$, duration of zero-order absorption; $F_{1}$, fraction absorbed through first-order absorption; $K_{\mathrm{a}}$, absorption rate constant; $A L A G_{1}$, lag time for first-order absorption; $C L / F$, apparent oral clearance; $V_{2} / F$, central volume; $V_{3} / F$, peripheral volume; $Q / F$, intercompartmental clearance. 
Table 3. Summary of final population PK parameters

\begin{tabular}{|c|c|c|c|c|c|c|}
\hline \multirow[b]{2}{*}{ Parameter } & \multicolumn{3}{|c|}{ MB1 } & \multicolumn{3}{|c|}{ MB2 } \\
\hline & Estimates & RSE (\%) & $\begin{array}{l}\text { Bootstrap median } \\
\qquad(95 \% \mathrm{Cl})\end{array}$ & Estimates & RSE (\%) & $\begin{array}{l}\text { Bootstrap median } \\
\qquad(95 \% \mathrm{Cl})\end{array}$ \\
\hline \multicolumn{7}{|l|}{ Structural model } \\
\hline CL/F (Period 1*) & 25.6 & 6.10 & $25.3(22.4-28.7)$ & 25.5 & 6.00 & $25.2(22.7-28.6)$ \\
\hline$C L$ change & - & - & - & 3.90 & 23.4 & $4.16(2.20-6.55)$ \\
\hline$V_{2} / F$ & 884 & 7.50 & $864(622-1,050)$ & 943 & 8.60 & $921(748-1,080)$ \\
\hline$V_{3} / F$ & 446 & 27.6 & $533(124-1,360)$ & 403 & 37.0 & $462(203-982)$ \\
\hline$Q / F$ & 63.6 & 21.2 & 70.4 (22.6-122) & 47.6 & 34.2 & $51.4(22.2-92.5)$ \\
\hline$K_{\mathrm{a}}$ & 0.874 & 16.1 & $0.835(0.453-1.55)$ & 0.991 & 17.6 & $0.959(0.588-1.44)$ \\
\hline$A L A G_{1}$ & 3.75 & 3.00 & $3.77(3.56-4.05)$ & 3.86 & 1.70 & 3.87 (3.73-4.08) \\
\hline$D_{2}$ & 3.31 & 9.10 & $3.35(2.91-4.56)$ & 3.42 & 5.10 & $3.44(3.03-3.94)$ \\
\hline$F_{1}\left(\right.$ Period $\left.1^{*}\right)$ & 0.458 & 10.7 & $0.461(0.333-0.597)$ & 0.405 & 14.3 & $0.410(0.313-0.530)$ \\
\hline$F_{1}($ Period 2$)$ & - & - & - & 0.468 & 9.80 & $0.474(0.391-0.563)$ \\
\hline$B I O$ & - & - & - & 1.30 & 3.50 & $1.30(1.20-1.41)$ \\
\hline \multicolumn{7}{|c|}{ Between-subject variability (CV\%, covariance for $\rho$ ) } \\
\hline$\omega_{\mathrm{CL}}$ & 29.9 & 30.4 & $29.3(20.4-39.2)$ & 29.8 & 28.8 & $28.6(20.5-37.2)$ \\
\hline$\omega_{\mathrm{V} 2}$ & 24.8 & 36.2 & $24.1(15.6-33.1)$ & 25.6 & 41.4 & $24.2(14.6-35.8)$ \\
\hline$\omega_{\mathrm{F} 1}$ & 32.1 & 44.0 & $30.4(18.5-51.6)$ & 33.4 & 55.3 & $30.7(17.6-52.1)$ \\
\hline$\omega_{\mathrm{BIO}}$ & - & - & - & 8.80 & 43.5 & $8.30(4.20-12.3)$ \\
\hline$\rho_{\mathrm{CL}-\mathrm{V} 2}$ & 0.857 & - & $0.872(0.581-0.999)$ & 0.802 & - & $0.800(0.464-0.946)$ \\
\hline \multicolumn{7}{|c|}{ Intra-individual variability (residual error) } \\
\hline$\sigma_{\text {Add }}$ & 0.408 & 5.20 & $0.401(0.357-0.442)$ & 0.456 & 5.60 & $0.451(0.397-0.499)$ \\
\hline
\end{tabular}

MB1, partial Model-based approach; MB2, full Model-based approach. RSE, relative standard errors; PK, pharmacokinetic; Cl, confidence interval; $C L / F$, clearance; $V_{2} / F$, central volume; $V_{3} / F$, peripheral volume; $Q / F$, inter-compartmental clearance; $K_{\mathrm{a}}$ absorption rate constant; $A L A G_{1}$, lag time for first-order absorption; $D_{2}$, duration of zero-order absorption; $F_{1}$, fraction absorbed through first-order absorption; $B I O$, total bioavailability of Period 2; $\omega_{\mathrm{CL}}$, between-subject variability of $C L ; \omega_{\mathrm{V} 2}$, between-subject variability of $V_{2}$; $\omega_{\mathrm{F} 1}$, between-subject variability of $F_{1} ; \omega_{\mathrm{BIO}}$, between-subject variability of $B I O ; \rho_{\mathrm{CL}-\mathrm{V}_{2}}$, correlation coefficient of $C L$ and $V_{2} ; \sigma_{\mathrm{Add}}$, additive error. *Results were rounded to 3 significant digits; Period 1 applies to MB2 only.

respectively, indicating DDI is not significant. The average $\mathrm{BE}$ results for all DDI evaluation methods are summarized in Table 4.

\section{DISCUSSION}

In this study, two types of model-based approach methods were assessed for DDI evaluation. Overall, both approaches with simulated data showed similar results using raw data. In all cases, including the conventional approach (NCA \& BE), $C_{\max }$ increased by about $13.9 \%-17.7 \%$, and $A U C_{\tau}$ increased by $10.5 \%-11.9 \%$ between periods. However, due to the small intra-subject variability of amlodipine [13], the PK parameter ratio's CI was very narrow, satisfying the classical BE limit. There may be some differences in the actual numbers depending on the method used, but such differences do not significantly change the likelihood of clinical DDI occurrence. Therefore, confidently all approaches properly evaluated the degree of DDI occurrence.

A model-based approach can have several advantages over the conventional method, which uses raw data only. For drugs with a long half-life, the possibility of DDI overestimation due to changes in concentration associated with reaching steady-state may be reduced; this was confirmed by comparing the results of the conventional approach and MB1. The conventional approach showed a larger ratio of $\mathrm{PK}$ parameters $\left(C_{\max }\right.$ and $\left.A U C_{\tau}\right)$ than MB1 because the simulated data corresponding to Period 2 in $\mathrm{MB1}$ reflected the steady-state concentration. At the time of study design, repeated administration is performed for a predicted period of steady-state achievement based on a known half-life. However, there is an uncertainty that the half-life may be longer than known in the subject population, and a long-term administration may be difficult to overcome this uncertainty for drugs with a long half-life [14]. Therefore, when evaluating clinical DDI with these drugs, using MB1 in addition to the conventional method may be sufficient to determine whether or not the DDI is overestimated in situations where steady-state is not achieved.

In the case of MB2, information on the change of each parameter was obtained from estimating model parameters that optimally described each period by using data from both periods. Although the role of the conventional approach and MB2 may be similar in that the overall $C_{\max }$ and $A U C_{\tau}$ show an increased pattern, the conventional approach has a limitation where it cannot be ascertained what mechanism is responsible for the change in the PK parameter [5,9]. According to the modeling results, it is highly likely that the increase in $C_{\max }$ was based on the increase in bioavailability $(\mathrm{BIO})$, indicating that the absorption of the drug in Period 2 increased by $30.0 \%$ compared to Period 1 . However, the actual increase in $C_{\max }$ and $A U C_{\tau}$ was not more significant than 
A
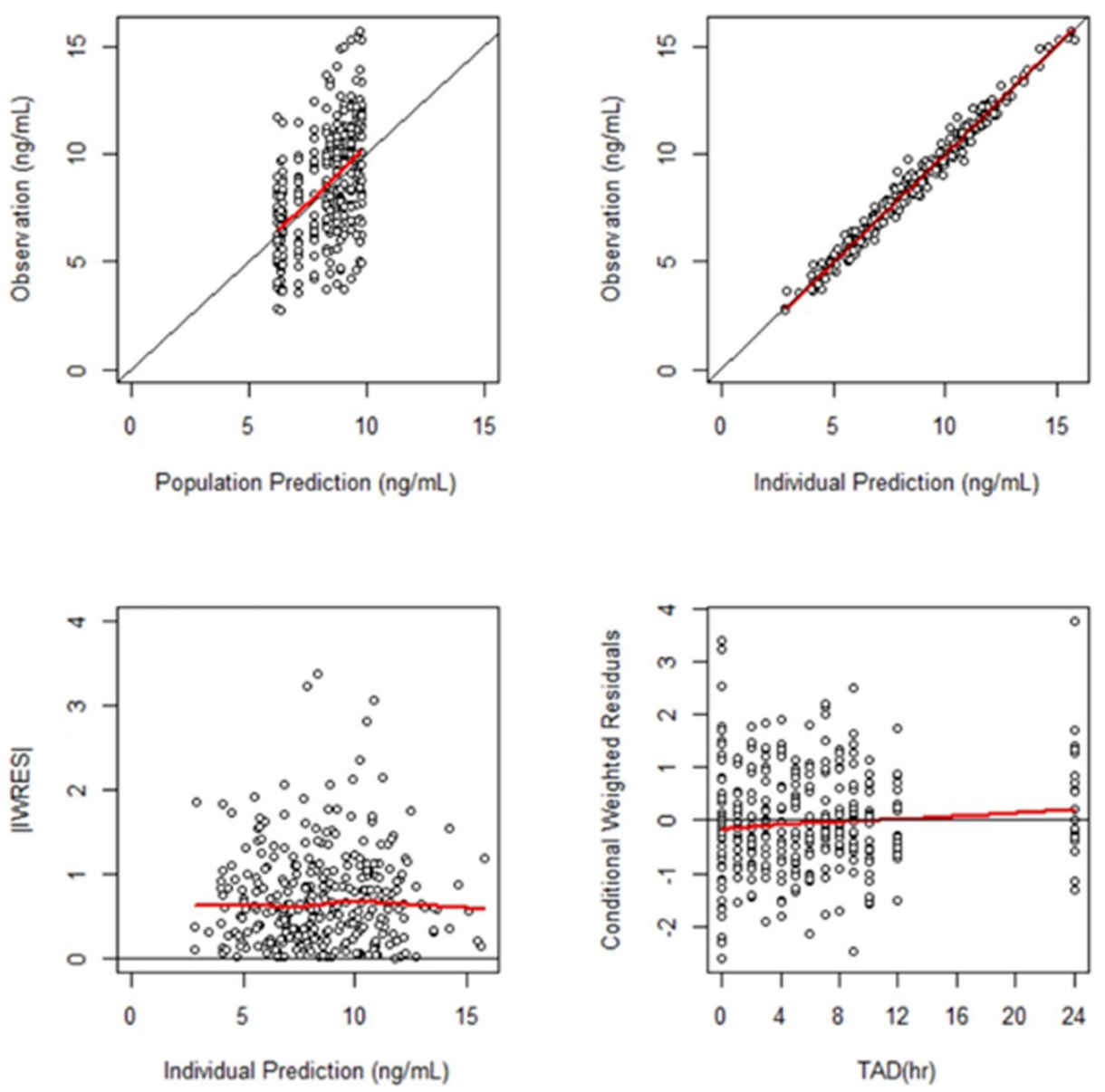

B

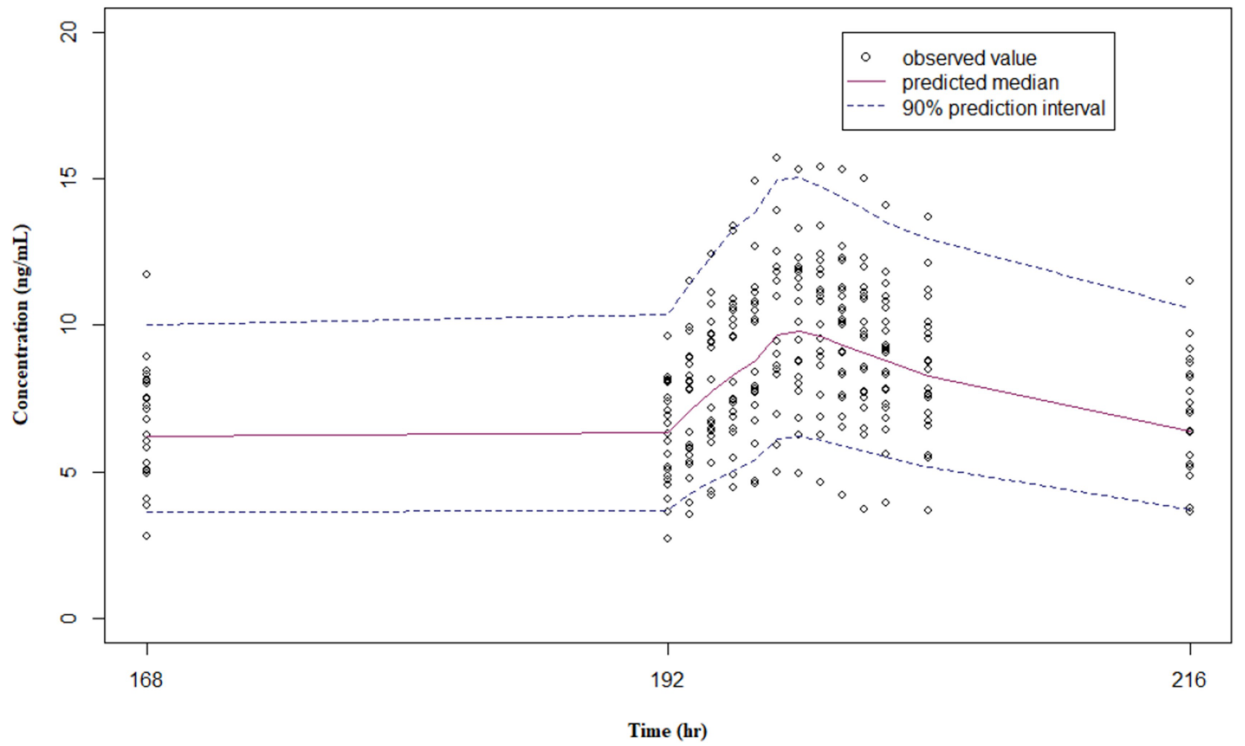

Fig. 3. Model outcomes of MB1. (A) Goodness-of-fit plots. (B) Visual predictive check. MB1, partial Model-based approach; TAD, time after dose; IWRES, individual weighted residuals. this because the increase in bioavailability was offset to some extent by $15.3 \%$ increased clearance (CL change); this is illustrated in Fig. 1, where Period 2's terminal slope of elimination is larger than that of Period 1.

The model-based approach is valuable because it provides additional information while helping to overcome limitations in 
A
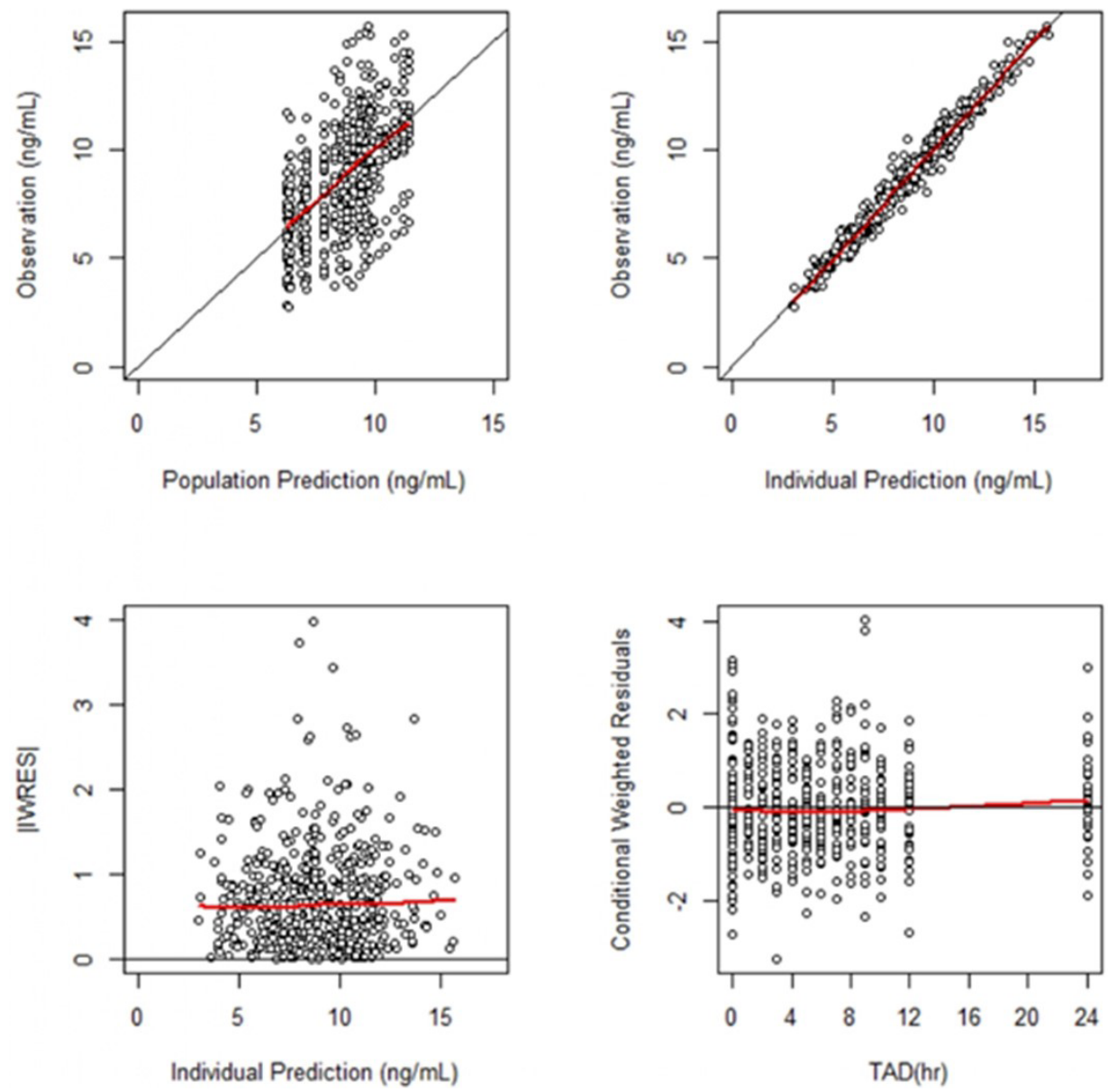

B

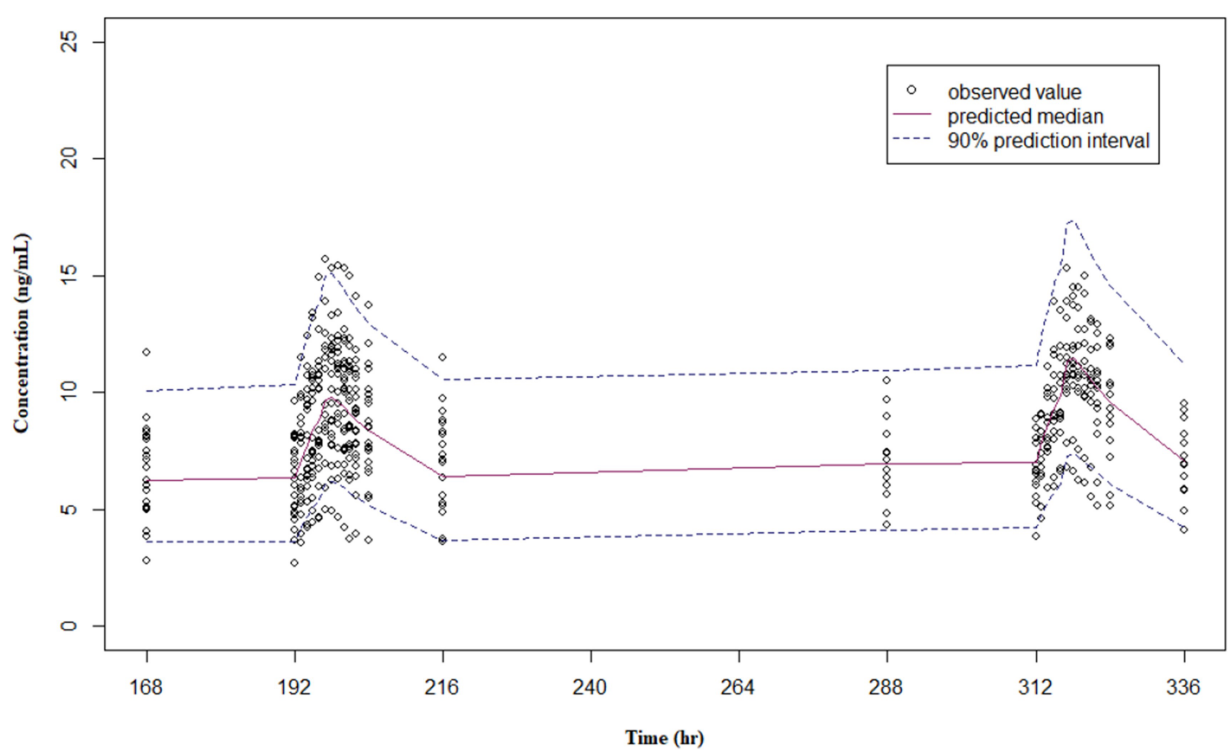

Fig. 4. Model outcomes of MB2. (A) Goodness-of-fit plots. (B) Visual predictive checks. MB2, full Model-based approach. TAD, time after dose; IWRES, individual weighted residuals.

clinical trial design. First, it is possible to predict $C_{\max }$ and $A U C_{\tau}$ without simulation through model parameter changes in two periods. Using $A U C_{\tau}$ as an example, the following calculation can be performed. Using the parameter estimates of $B I O$ and $C L$ from the model, $A U C$ was calculated by the equations below: 
Table 4. Summary table for BE limit with average geometric mean ratios and $90 \%$ confidence intervals (lower and upper) of DDI evaluation methods $(n=1,000)$

\begin{tabular}{|c|c|c|c|c|c|c|}
\hline \multirow{2}{*}{ Estimates } & \multicolumn{3}{|c|}{$C_{\max }$} & \multicolumn{3}{|c|}{$A \cup C_{\mathrm{T}}$} \\
\hline & NCA \& BE & MB1 & MB2 & $\mathrm{NCA} \& \mathrm{BE}$ & MB1 & MB2 \\
\hline Lower & 1.127 & $\begin{array}{c}1.097 \\
(1.079-1.114)\end{array}$ & $\begin{array}{c}1.092 \\
(1.088-1.095)\end{array}$ & 1.068 & $\begin{array}{c}1.048 \\
(1.036-1.059)\end{array}$ & $\begin{array}{c}1.064 \\
(1.063-1.066)\end{array}$ \\
\hline Point estimate & 1.177 & $\begin{array}{c}1.149 \\
(1.130-1.167)\end{array}$ & $\begin{array}{c}1.139 \\
(1.134-1.143)\end{array}$ & 1.119 & $\begin{array}{c}1.105 \\
(1.092-1.118)\end{array}$ & $\begin{array}{c}1.116 \\
(1.113-1.118)\end{array}$ \\
\hline Upper & 1.229 & $\begin{array}{c}1.203 \\
(1.179-1.227)\end{array}$ & $\begin{array}{c}1.188 \\
(1.181-1.195)\end{array}$ & 1.174 & $\begin{array}{c}1.166 \\
(1.147-1.186)\end{array}$ & $\begin{array}{c}1.170 \\
(1.165-1.175)\end{array}$ \\
\hline Percentage satisfied (\%) & - & 100 & 100 & - & 100 & 100 \\
\hline
\end{tabular}

MB1, partial Model-based approach; MB2, full Model-based approach. BE, bioequivalence; DDI, drug-drug interaction; NCA, noncompartmental analysis.

$$
A U C=F \cdot D O S E / C L
$$

Assuming the same dose (DOSE) was given, the relative exposure was described by:

$$
A U C_{\text {period2 }} / A U C_{\text {period1 }}
$$

The point estimate was 1.124 , and the $90 \%$ confidence interval was 1.089-1.154, similar to the results above. Second, precise changes in parameters provide an opportunity to assess whether such trends in DDI can be explained by the known effects of concomitant drugs on drug transporters or metabolic enzymes [15]. Finally, this approach provides a confidence limit in addition to the point estimate; this can be used as an indicator of the likelihood that the same results can be derived during repeated simulations [9]. The result confirmed that the $90 \% \mathrm{CI}$ remained within $0.8-1.25$, and accordingly, all met the classical BE limit.

Model-based comparative PK evaluations are not yet widely used, and results have not been published in many cases. In this study, several advantages of the model-based approach were demonstrated, and possible application methods were proposed.

With similar experiences accumulated from comparative $\mathrm{PK}$ studies on drugs of various characteristics, it is expected that the advantages of the model-based approach compared to the classical method can be appropriately utilized according to the situation and purpose.

\section{ACKNOWLEDGEMENTS}

The authors wish to acknowledge the financial support of the Catholic Medical Center Research Foundation made in the program year of 2016 .

\section{CONFLICTS OF INTEREST}

The authors declare no conflicts of interest.

\section{REFERENCES}

1. Düsing R. Optimizing blood pressure control through the use of fixed combinations. Vasc Health Risk Manag. 2010;6:321-325.

2. Lin CP, Tung YC, Hsiao FC, Yang CH, Kao YW, Lin YS, Chu YC, $\mathrm{Chu} \mathrm{PH}$. Fixed-dose combination of amlodipine and atorvastatin improves clinical outcomes in patients with concomitant hypertension and dyslipidemia. J Clin Hypertens (Greenwich). 2020;22:18461853.

3. Simons LA, Chung E, Ortiz M. Long-term persistence with singlepill, fixed-dose combination therapy versus two pills of amlodipine and perindopril for hypertension: Australian experience. Curr Med Res Opin. 2017;33:1783-1787.

4. U.S. Food and Drug Administration. Amlodipine prescribing information [Internet]. Silver Spring: U.S. Food and Drug Administration, 2020 [cited 2020 Nov 20]. Available from: https://www. accessdata.fda.gov/drugsatfda_docs/label/2019/211340s000lbl.pdf.

5. U.S. Food and Drug Administration. Clinical drug interaction studies - cytochrome P450 enzyme- and transporter-mediated drug interactions guidance for industry [Internet]. Silver Spring: U.S. Food and Drug Administration, 2020 [cited 2020 Nov 20]. Available from: https://www.fda.gov/media/134581/download.

6. Lehr T, Staab A, Trommeshauser D, Schaefer HG, Kloft C. Semimechanistic population pharmacokinetic drug-drug interaction modelling of a long half-life substrate and itraconazole. Clin Pharmacokinet. 2010;49:53-66.

7. Svensson EM, Acharya C, Clauson B, Dooley KE, Karlsson MO. Pharmacokinetic interactions for drugs with a long half-life-evidence for the need of model-based analysis. AAPS J. 2016;18:171-179.

8. Choi S, Jeon S, Yim DS, Han S. Contribution of trough concentration data in the evaluation of multiple-dose pharmacokinetics for drugs with delayed distributional equilibrium and long half-life. Drug Des Devel Ther. 2020;14:811-821.

9. Seng Yue C, Ozdin D, Selber-Hnatiw S, Ducharme MP. Opportunities and challenges related to the implementation of model-based bioequivalence criteria. Clin Pharmacol Ther. 2019;105:350-362.

10. Kim H, Han S, Cho YS, Yoon SK, Bae KS. Development of R packages: 'NonCompart' and 'ncar' for noncompartmental analysis (NCA). Transl Clin Pharmacol. 2018;26:10-15.

11. U.S. Food and Drug Administration. Draft guidance for industry: bioequivalence studies with pharmacokinetic endpoints for drugs 
submitted under an ANDA. Silver Spring: U.S. Food and Drug Administration, 2013 [cited 2020 Nov 20]. Available from: https://www. fdanews.com/ext/resources/files/12/12-05-13-ANDAGuidance.pdf.

12. Heo YA, Holford N, Kim Y, Son M, Park K. Quantitative model for the blood pressure-lowering interaction of valsartan and amlodipine. Br J Clin Pharmacol. 2016;82:1557-1567.

13. Wang T, Wang Y, Lin S, Fang L, Lou S, Zhao D, Zhu J, Yang Q, Wang Y. Evaluation of pharmacokinetics and safety with bioequivalence of Amlodipine in healthy Chinese volunteers: Bioequivalence
Study Findings. J Clin Lab Anal. 2020;34:e23228.

14. Maganti L, Panebianco DL, Maes AL. Evaluation of methods for estimating time to steady state with examples from phase 1 studies. AAPS J. 2008;10:141-147.

15. Foster DM. Noncompartmental versus compartmental approaches to pharmacokinetic analysis. In: Atkinson AJ Jr, Abernethy DR, Daniels CE, Dedrick RL, Markey SP, editors. Principles of clinical pharmacology. Amsterdam: Elsevier; 2007. p.89-105. 